J. Gastroenterol. Hepatol. Erkr. 2019 · 17:18-24 https://doi.org/10.1007/s41971-019-0045-2

Online publiziert: 25. Februar 2019

(c) Der/die Autor(en) 2019

\section{Christian Müller}

Univ.-Klinik für Innere Medizin III, Klin. Abteilung Gastroenterologie und Hepatologie, Medizinische Universität Wien, Wien, Österreich

\title{
Operationsrisiko bei chronischen Lebererkrankungen
}

\section{Einleitung}

Patienten mit schweren Lebererkrankungen weisen im Vergleich zu Patienten ohne Leberprobleme ein erhöhtes Morbiditäts- und Mortalitätsrisiko bei chirurgischen Eingriffen auf [1-3].

Das erhöhte Risiko setzt sich aus mehreren Einzelfaktoren zusammen: So ist oft das rein chirurgische Risiko aufgrund erschwerter chirurgischer Bedingungen erhöht (portale Hypertension, Kollateralenbildungen), von anästhesiologischer Seite können relevante Veränderungen des Metabolismus von Anästhetika und eine instabile perioperative Hämodynamik von Bedeutung sein, aus internistischer Sicht erhöhen Infektionen, Nierenfunktionseinschränkung und hepatische Enzephalopathie das Gesamtrisiko.

Beica. $10 \%$ der Patienten mit Lebererkrankung muss aus den unterschiedlichsten Gründen innerhalb der letzten beiden Lebensjahre eine Operation durchgeführt werden [4], somit ist die klinische präoperative Risikoeinschätzung eine häufige Fragestellung. Da vielfach eine beträchtliche Unsicherheit bezüglich des vorhandenen Operationsrisikos besteht, werden bei vielen Patienten mit a priori niedrigem Operationsrisiko unnötige präoperative Untersuchungen veranlasst, auf der anderen Seite werden $\mathrm{Pa}$ tienten mit beträchtlichem operativem Risiko vermeidbaren Operationen ausgesetzt. Die zur Risikobestimmung notwendige Datenlage ist jedoch insgesamt als nur mangelhaft zu bezeichnen - sie stammt praktisch ausschließlich aus re-

Aktualisierung von: Müller Ch (2004) Leber und Operationsrisiko. J Gastroenterol Hepatol Erkr 2(2):36-39 trospektiven Fallserien, sehr oft aus den 80er- und 90er-Jahren des vorangegangenen Jahrhunderts. Die seither beträchtlichen Fortschritte der chirurgischen und anästhesiologischen Techniken und des heute flächendeckend zur Verfügung stehenden postoperativen intensivmedizinischen Monitorings lassen vermuten, dass das Operationsrisiko für Patienten mit Lebererkrankung - obwohl nach wie vor in bestimmten Situationen gegenüber Nichtlebererkrankten erhöht - deutlich gesunken ist.

\section{Tools zur Risikoabschätzung}

\section{Leberwerte}

Die „Leberwerte“ sind Hilfsmittel zur Erkennung einer Hepatopathie und ihrer ätiologischen Einordnung; sie eignen sich weniger zur Abschätzung des operativen Risikos ([5]; • Tab. 1).

Leberwerte ermöglichen zusammen mit den klinischen Zeichen und Symptomen die Diagnose "Lebererkrankung“ und die Zuordnung in eine cholestatische oder mehr hepatische Verlaufsform.

\section{Quantitative Leberfunktionstests}

Quantitative Leberfunktionstests beruhen auf der Bestimmung der Verstoffwechselungsrate und Eliminationshalbwertszeit exogen zugeführter Xenobiotika. Dazu gehören die Indocyaningrünhalbwertszeit, der Monoethylglycinexylidid(MEGX)-Lidocaintest und der Aminopyrinatemtest. Es besteht eine gute Korrelation dieser Parameter zum Ausmaß der bestehenden Leberschädigung $[6,7]$. Die Teste sind jedoch in ihrer Durchführung aufwendig und kei- ner weist einen klaren Vorteil gegenüber den weit einfacheren Scoringsystemen der Leberfunktion auf, sodass sie in der klinischen Routine nur selten eingesetzt werden.

\section{Scoringsysteme}

Die Leber führt mit ihren Teilaufgaben Synthese, Gallenproduktion und Detoxifikation eine Vielzahl von Funktionen aus, die in unterschiedlichem Ausmaß bei Patienten mit chronischer Lebererkrankung beeinträchtigt sind. Ein einzelner Parameter, der das Gesamtausmaß der erhaltenen Leberfunktion beurteilen könnte, existiert nicht. Es müssen daher die Teilfunktionen getrennt beurteilt und zu einem Gesamtpunktescore zusammengefasst werden, der zum Überleben und auch zum Mortalitätsrisiko bei Operationen korreliert. Das älteste und bekannteste dieser Scoringsysteme ist die von Child und Turcotte im Jahr 1964 [8] vorgeschlagene und von Pugh [9] 1973 modifizierte Child-PughKlassifikation. Child und Turcotte entwickelten den Score zur Risikoabschätzung der zu ihrer Zeit häufig durchgeführten portokavalen Shuntoperation, er ist jedoch auch zur Risikoabschätzung bei anderen chirurgischen Eingriffen und auch zur allgemeinen Prognoseabschätzung bei Patienten mit Leberzirrhose geeignet ([10]; • Tab. 2). Im ursprünglichen Scoringsystem erfolgte eine subjektive Einschätzung des Therapieansprechens bei Aszites, des Ernährungszustands und der Enzephalopathie. In der Modifikation nach Pugh wurde der Ernährungszustand durch die PTZ ersetzt [9], für die Enzephalopathie-Einschätzung werden heute meistens die West- 


\begin{tabular}{|c|c|}
\hline \multirow{4}{*}{$\begin{array}{l}\text { 1. Hepatozelluläre } \\
\text { Integrität }\end{array}$} & GOT (AST) \\
\hline & GPT (ALT) \\
\hline & LDH \\
\hline & Ferritin \\
\hline \multirow[t]{2}{*}{ 2. Biliäre Integrität } & $\begin{array}{l}\text { Alkalische Phospha- } \\
\text { tase }\end{array}$ \\
\hline & Y-GT \\
\hline \multirow[t]{4}{*}{ 3. Syntheseleistung } & Albumin $^{\mathrm{a}}$ \\
\hline & Cholinesterase $\mathrm{e}^{\mathrm{a}, \mathrm{b}}$ \\
\hline & PTZ, INR \\
\hline & Fibrinogen $^{c}$ \\
\hline \multirow[t]{2}{*}{$\begin{array}{l}\text { 4. Anionentransport- } \\
\text { störung }\end{array}$} & $\begin{array}{l}\text { Bilirubin (direkt, } \\
\text { indirekt) }\end{array}$ \\
\hline & Gallensäuren \\
\hline $\begin{array}{l}\text { 5. Detoxifikations- } \\
\text { störung }\end{array}$ & Ammoniak \\
\hline \multicolumn{2}{|c|}{$\begin{array}{l}\text { Negativer Akute-Phase-Parameter. Nur } \\
\text { als Syntheseparameter verwendbar, wenn } \\
\text { keine Akute-Phase-Reaktion vorliegt } \\
\text { 'Auch abhängig vom Ernährungszustand } \\
\text { 'Positiver Akute-Phase-Parameter. In der } \\
\text { Akutphase erhöht, daher in diesem Fall nicht } \\
\text { als Syntheseparameter heranziehbar } \\
\text { AST Aspartat-Aminotransferase, GOTGlutamat- } \\
\text { Oxalacetat-Transaminase, ALT Alanin-Ami- } \\
\text { notransferase, GPT Glutamat-Pyruvat-Tran- } \\
\text { saminase, LDH Lactate dehydrogenase, } \\
\text { Y-GT Gamma-Glutamyl Transpeptidase, } \\
\text { PTZ Prothrombinzeit, Thromboplastinzeit, } \\
\text { INR International Normalized Ratio }\end{array}$} \\
\hline
\end{tabular}

\section{Tab. 3 MELD-Score}

$3,8 \times \ln ($ Bilirubin in $\mathrm{mg} / \mathrm{dl})+$

$11,2 \times \ln ($ INR $)+$

$9,6 \times \ln$ (Kreatinin in $\mathrm{mg} / \mathrm{dl}$ )

[15]

MELD "Mayo model for endstage liver

disease", INR

haven-Kriterien zur qualitativen Evaluierung der hepatischen Enzephalopathie eingesetzt. Dem Child-Pugh-Score liegt kein prospektiv evaluiertes mathematisches Modell zugrunde, er wurde von den Autoren aus ihrer klinischen Erfahrung und ihrer Intuition entwickelt. Aufgrund seiner leichten Handhabbarkeit ist er auch heute noch immer das am häufigsten verwendete Scoringsystem, obwohl der MELD-Score dem Child-Pugh-Score auch in der chirurgischen Risikoabschätzung überlegen erscheint [11].

DerMELD(„Mayo model for endstage liver disease")-Score wurde ursprünglich

\begin{tabular}{|c|c|c|c|c|}
\hline Parameter & 1 Punkt & 2 Punkte & 3 Punkte & Patient Punkte \\
\hline Bilirubin & $<1,0 \mathrm{mg} / \mathrm{dl}$ & $1-3 \mathrm{mg} / \mathrm{dl}$ & $>3 \mathrm{mg} / \mathrm{dl}$ & - \\
\hline Albumin & $>3,5 \mathrm{~g} / \mathrm{dl}$ & $3,0-3,5 \mathrm{~g} / \mathrm{dl}$ & $<3,0 \mathrm{~g} / \mathrm{dl}$ & - \\
\hline $\begin{array}{l}\text { PTZ oder } \\
\text { INR }\end{array}$ & $\begin{array}{l}>70 \% \\
<1,7\end{array}$ & $\begin{array}{l}40-70 \% \\
1,7-2,3\end{array}$ & $\begin{array}{l}<40 \% \\
>2,3\end{array}$ & - \\
\hline $\begin{array}{l}\text { Enzephalo- } \\
\text { pathiegrad }\end{array}$ & 0 & $1-2$ & $3-4$ & - \\
\hline Aszites & Kein Aszites & $\begin{array}{l}\text { Therapierbar in- } \\
\text { nerhalb } 1 \text { Woche }\end{array}$ & Therapierefraktär & - \\
\hline- & $\begin{array}{l}\text { Ein-Jahres- } \\
\text { Überleben }\end{array}$ & $\begin{array}{l}\text { Zwei-Jahres- } \\
\text { Überleben }\end{array}$ & - & Summe: \\
\hline Child-Pugh A & $100 \%$ & $85 \%$ & Child-Pugh A & $5-6$ \\
\hline Child-Pugh B & $80 \%$ & $60 \%$ & Child-Pugh B & $7-9$ \\
\hline Child-Pugh B & $45 \%$ & $35 \%$ & Child-Pugh C & $10-15$ \\
\hline
\end{tabular}

zur Prognoseabschätzung von Patienten vor TIPS-Implantation entwickelt [12, 13]. Der MELD-Score ist ein prospektives evaluiertes Prognosemodell, für das aus einer großen Anzahl klinischer Parameter durch Multivarianzanalyse und logistische Regression 4 mit der Prognose signifikant assoziierte Parameter identifiziert wurden: Bilirubin, Gerinnung (INR), Kreatinin und Ätiologie der Lebererkrankung (• Tab. 3). In der heute allgemein benutzten Variante des MELD-Scores wird der Faktor Ätiologie der Lebererkrankung nicht mehr verwendet, da dieser die Genauigkeit des Modells bezüglich der Vorhersagekraft des Überlebens nicht wesentlich verbessert. Der Vorteil des MELD-Scores liegt in der Verwendung von Parametern, die gemessen werden können und nicht einer subjektiven Fehleinschätzung unterliegen können. Er korreliert ausgezeichnet mit der Mortalität von Patienten mit Lebererkrankungen ([14]; • Abb. 1). Der MELD-Score ist etwas aufwendig zu berechnen und somit nicht unmittelbar am Krankenbett abschätzbar. Im Internet sind mehrere MELD-Score-Rechner abrufbar, z.B. unter https://www. mayoclinic.org/medical-professionals/ transplant-medicine/calculators/meldmodel/itt-20434705.

Der MELD-Score wurde in prospektiven Studien zur Abschätzung des Operationsrisikos bei Leberzirrhose untersucht. Bei Patienten mit Leberzirrhose war der MELD-Score der beste Prädiktor für die 30- und 90-Tage-Mortalität und zeigte eine lineare Korrelation [16].
Andere Studien verglichen den ChildPugh-Score mit dem MELD-Score und zeigten häufig, wenn auch nicht immer, eine Überlegenheit des MELD-Scores gegenüber dem Child-Pugh-Score [17-20]. Hanje et al. [21] halten Patienten mit Leberzirrhose und einem MELD-Score unter 10 für geeignet für eine elektive chirurgische Intervention. Bei einem MELDScore zwischen 10 und 15 ist eine Operation unter erhöhtem Risiko möglich. Bei einem MELD-Score über 15 sollte vor einer elektiven chirurgischen Intervention Abstand genommen werden. Basierend auf dem MELD-Score, der ASAKlassifikation und dem Patientenalter ist ein postoperativer Mortalitätsrisikorechner bei Patienten mit Leberzirrhose aufrufbar [16, 22].

\section{Bewertung des Operationsrisikos}

Die Frage des Operationsrisikos bei Patienten mit Lebererkrankung soll anhand folgender klinischer Situationen behandelt werden.

\section{Patient ohne bekannte Leberer- krankung mit zufällig entdeckten erhöhten „Leberwerten"}

Diese Situation kommt mit einer Häufigkeit von ca. 1:300 bis 1:700 bei Operationskandidaten ohne vorbekannte Lebererkrankung vor [23, 24]. Die Erhöhung nur eines Einzelwerts $(\gamma-G T$, alkalische Phosphatase, Transaminasen) hat keine fassbare Erhöhung des chi- 
rurgischen Risikos zur Folge [3]. In der nichtinvasiven Abklärung hilft eine nochmalige Kontrolle nach Alkoholkarenz oder das Absetzen medikamentöser Therapien. Isolierte Erhöhungen von einzelnen Leberwerten sind häufig auch bei nichthepatologischen Erkrankungen zu beobachten (Übergewicht, Diabetes mellitus, kardiale Dekompensation, Kollagenosen, Vaskulitiden, Sarkoidose). Sind hingegen mehrere "Leberwerte“ bei Patienten ohne vorbekannte Lebererkrankung erhöht, ist eine bisher nicht erkannte hepatale Grunderkrankung möglich. Bei asymptomatischen Patienten ohne akute Lebererkrankung kann das Operationsrisiko anhand der Child-Pugh-Klassifikation orientierend abgeschätzt werden. Da es sich um asymptomatische Patienten handelt, kann als „worst case scenario“ maximal eine kompensierte Leberzirrhose im Stadium Child-Pugh A angenommen werden. Da für Child-Pugh-A-Leberzirrhosen selbst kein wesentlich erhöhtes Operationsrisiko angenommen werden muss, kann unter Voraussetzung einer korrekten Operationsindikation eine elektive Operation auch ohne weitere Abklärung der Lebererkrankung (Leberbiopsie) durchgeführt werden [3].

\section{Patienten mit bekannter stabiler Lebererkrankung}

Patienten mit chronischer Virushepatitis (Hepatitis B, Hepatitis C) weisen keine erhöhte präoperative Mortalität im Vergleich zu nichthepatal erkrankten Patienten auf [25]. Patienten mit Steatosis hepatis weisen eine gegenüber Lebergesunden nicht erhöhte operative Mortalität auf, lediglich bei hepatischen Resektionen ist die Mortalität mit dem histologischen Ausmaß der Steatose verknüpft. Patienten mit mehr als $30 \%$ Steatose weisen eine Mortalität von $14 \%$ auf gegenüber $3 \%$ Mortalität bei Patienten mit weniger als $5 \%$ hepatalem Fett [26]. Liegt eine nichtalkoholische Steatohepatitis (NASH) vor, ist die Morbidität nach hepatischer Resektion gegenüber Patienten mit reiner Steatose erhöht [27]. Obwohl NASH eine häufige Komplikation bei Patienten mit morbider Adipositas ist und in diesen Patienten auch häu-

J. Gastroenterol. Hepatol. Erkr. 2019 · 17:18-24 https://doi.org/10.1007/s41971-019-0045-2 c) Der/die Autor(en) 2019

\section{Müller \\ Operationsrisiko bei chronischen Lebererkrankungen}

\section{Zusammenfassung}

Erhöhte "Leberwerte" stellen präoperativ ein häufiges klinisches Problem unterschiedlicher Signifikanz dar. Prognostisch stellen erhöhte Einzelwerte keine signifikante Veränderung dar, sind mehrere Leberwerte erhöht, sollte präoperativ zumindest eine Abklärung mittels Virusserologie und Ultraschall erfolgen. Bei Patienten ohne klinische Symptomatik einer Lebererkrankung, bei Patienten mit chronischer Hepatitis ohne Leberzirrhose und bei Patienten mit Leberzirrhose im Stadium Child-Pugh A ist bei extrahepatischen Operationen kein erhöhtes Operationsrisiko zu erwarten. Symptomatische Patienten mit Lebererkrankung (Child-Pugh-B- und Child-
Pugh-C-Leberzirrhose) weisen ein deutlich erhöhtes Operationsrisiko auf, bei ChildPugh-C-Zirrhose ist eine elektive Operation aufgrund der beträchtlichen Mortalität (bis $60 \%$ ) meistens als Kontraindikation zu sehen, bei Child-Pugh-B-Zirrhosen kann nur ein problemorientiertes postoperatives Management Morbidität und Mortalität senken.

\section{Schlüsselwörter}

Quantitative Leberfunktionstests · Scoring Systeme · Isolierte Gamma-GT Erhöhung . Aszites · Ikterus

\section{Surgical Risk in Chronic Liver Diseses}

\section{Abstract}

Elevated "liver function tests" are often seen in patients undergoing surgery. Very often the significance of this finding is unclear and the urgency of a diagnostic work-up depends on the context of presentation and planned operative procedure. Elevation of one liver function test is most often not significant, while elevation of several liver function tests should prompt a diagnostic work-up for chronic viral hepatitis and a morphologic examination of the liver and gall duct by ultrasound. Patients with chronic liver disease without liver cirrhosis and patients with liver cirrhosis Child-Pugh A class do not have an increased operative risk, whereas the operative risk of Child-Pugh C cirrhosis is substantial and usually seen as a contraindication for elective surgery in those patients. Operations in patients with Child-Pugh B cirrhosis can be performed but require meticulous peri- and postoperative observation and intensive management.

\section{Keywords}

Quantitative liver function probes · Scoring systems · Increase Gamma-GT · Ascites . Jaundice figer eine NASH-Zirrhose vorliegt, können Patienten mit NASH-Zirrhose einem bariatrisch-chirurgischen Eingriff zugeführt werden [28]. Patienten mit einer gut eingestellten Autoimmunhepatitis unter immunsuppressiver Therapie mit Kortison und/oder Azathioprin sind geeignete Kandidaten für elektive chirurgische Eingriffe. Auch eine Lebersegmentresektion in einer Child-Pugh-A-Leberzirrhose ist gegenüber einer Lebersegmentresektion in einer nichtzirrhotischen Leber bei sorgfältiger Patientenselektion nur mit einer nicht signifikant erhöhten intraund postoperativen Mortalität verknüpft [29-31]. Geeignete Kandidaten für eine operative Resektion eines hepatozellulären Karzinomknotens sind Patienten mit Child-Pugh-A-Zirrhose, bei denen zusätzlich weder das Bilirubin über den Normbereich erhöht ist noch eine klinisch signifikante portale Hypertension (HPVG > $10 \mathrm{~mm} \mathrm{Hg}$ ) vorliegt [32].

\section{Symptomatischer Patient mit Lebererkrankung}

Hierbei handelt es sich meist um Patienten mit mechanischem Ikterus, manchmal wird auch eine akute Hepatitis unter einer anderen Verdachtsdiagnose einer Operation zugeführt. Auch Patienten mit bekannter dekompensierter Leberzirrhose fallen in diese Kategorie. Symptomatische Patienten mit Lebererkrankung weisen ein erhöhtes OP-Risiko auf 
Hier steht eine Anzeige.

\section{曾 Springer}




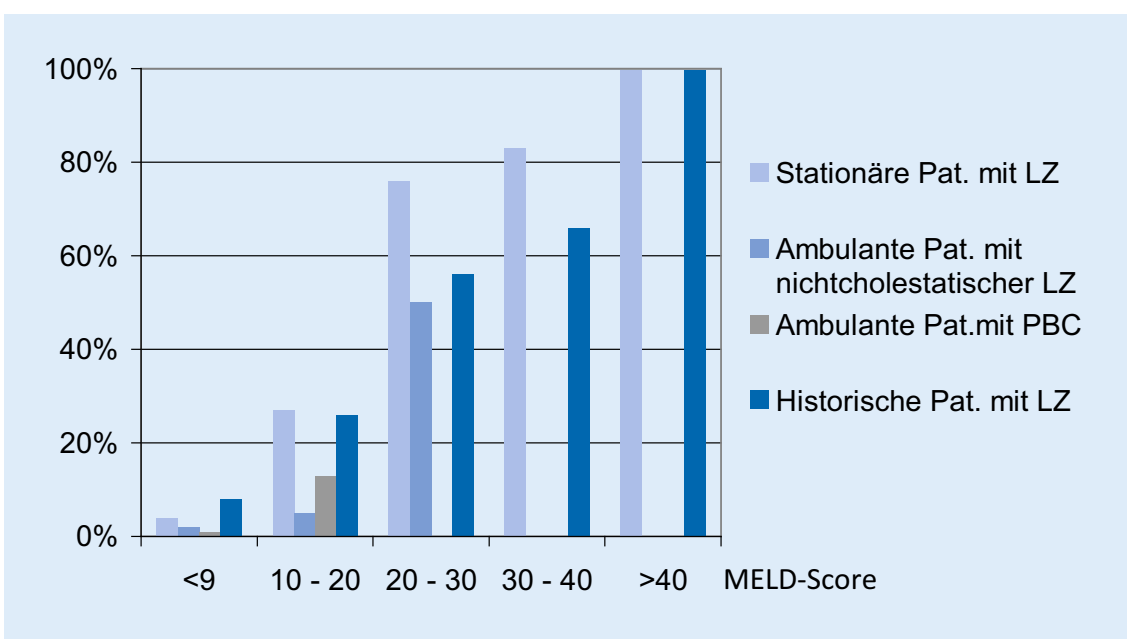

Abb. 1 Drei-Monate-Mortalität nach MELD-Score [13]. MELD „Mayo model for endstage liver disease"-Score

[8]. Zwei Studien aus dem Jahre 1984 [33] und 13 Jahre später 1997 [34] zeigen bei abdominellen offenen Operationen bei Child-Pugh-C- (ca. 70-80\%) und bei Child-Pugh-B-Zirrhose (ca. 30\%) eine praktisch unveränderte Mortalität über die Jahre. Eine bei Child-Pugh-C-Zirrhose-Patienten durchgeführte offene Cholezystektomie weist eine 30-Tage-Mortalität nach der Operation von ca. $80 \%$ auf, bei Child-Pugh A/B von ca. $9 \%$ [35]. Seit Einführung der laparoskopischen Cholezystektomie haben sich diese Zahlen deutlich verbessert, eine 2002 veröffentlichte Studie [36] zeigt eine Mortalität bei Leberzirrhose von lediglich $0,9 \%$; diese ist jedoch immer noch signifikant erhöht gegenüber den $0,01 \%$ bei nicht an Leberzirrhose erkrankten Patienten [37-39].

Eine laparoskopische Cholezystektomie kann bei Patienten mit Leberzirrhose Child-Pugh A oder B oder einem MELDScore bis zu 13 mit einer akzeptablen geringen Mortalität durchgeführt werden [40]. Eine offen durchgeführte kolorektale Chirurgie (wegen Divertikel oder kolorektaler Tumoren) ist mit Mortalitätsraten bis $26 \%$ vergesellschaftet [41, 42]. Auch hier könnte eine laparoskopische chirurgische Vorgangsweise, wenn krankheitsstadiengerecht möglich, eine Reduktion der Mortalität aufweisen.

Eine spezielle Situation stellen Patienten mit mechanischem Ikterus dar. Sie sind für eine Reihe von peri- und postoperativen Komplikationen, wie Infektionen, Stressulzera, disseminierte intravaskuläre Koagulation, Wunddehiszenz und Nierenversagen, anfällig. Die perioperative Mortalität dieser nichtzirrhotischen Patienten mit mechanischem Ikterus lag bei 9\% [43]. Eine Multivariatanalyse zeigte, dass ein Hämatokrit unter $30 \%$, ein Bilirubin über $11 \mathrm{mg} \%$ und eine maligne Natur der Obstruktion unabhängige Risikofaktoren darstellen. Bei Vorhandensein aller 3 Faktoren war die 30-Tage-Mortalität $60 \%$, wohingegen bei der Präsenz nur eines Faktors eine nur 5\%ige Mortalität zu verzeichnen war. Diese Hochrisikosituation wurde durch verschiedene präinterventionelle Eingriffe zu verändern versucht. Bei Vorhandensein eines malignen Verschlusses konnte jedoch weder für die präoperativ perkutane [44] noch für die endoskopisch transpapillare Drainage [45, 46] eine Reduktion der Mortalität gezeigt werden. Eine perioperative breitspektrumantibiotische Therapie führt zwar $\mathrm{zu}$ einer Reduktion perioperativer Infektionen, hat jedoch keinen Einfluss auf die Mortalität [47], die besonders durch postoperatives Nierenversagen beeinflusst wird [48].

Bei Vorliegen eines steinbedingten Gallengangsverschlusses ist die Situation jedoch gänzlich anders. Hier konnte überzeugend gezeigt werden, dass der Einsatz einer endoskopisch geführten Drainage und intravenöser Antibiotika mit einer Reduktion von Morbidität und Mortalität assoziiert ist [49].
Symptomatische Patienten mit Leberzirrhose wiesen in der Vergangenheit bei Resektion eines Lebertumors eine inakzeptable hohe Mortalität ( $>50 \%)$ auf; auch heute noch ist diese sehr hoch und für Child-Pugh-B- und -C-Patienten keine Option [50]. In den letzten Jahren ist die peri- und postoperative Mortalität deutlich gesunken und weist Mortalitätsraten von $8 \%$ für ausgedehnte Resektionen und 3,4\% für eingeschränkte Resektionen auf [31]. Eine deutlich verbesserte chirurgische Technik (minimalinvasive Resektion), verbunden mit intensiver postoperativer Überwachung und besserer Selektion von potenziellen Resektionskandidaten haben eine weitere Verbesserung erbracht [51]. So wird eine postoperative Mortalität von nur 1,2\% erreicht, wenn kein Leberversagen nach Resektion, definiert als INR $>1,7$ (entspricht PTZ $<50 \%$ ) und Bilirubin $>2,9 \mathrm{mg} / \mathrm{dl}$ (entspricht $50 \mu \mathrm{mol} / \mathrm{l}$ ), auftritt. Eine klinisch signifikante portale Hypertension (Splenomegalie mit Plättchen $<100.000 /$ ml oder Ösophagusvarizen) ist auch gegenwärtig noch mit einer hohen postoperativen Morbidität und Mortalität verknüpft [32]. Dementsprechend empfehlen die Leitlinien der Europäischen Lebergesellschaft zur Resektion des Hepatozellulären Karzinoms [52], eine Resektion bei singulären HCC-Knoten in Child-Pugh-A-Leberzirrhose mit ausgezeichneter Leberfunktion (normales Bilirubin) und klinisch nichtsignifikanter portaler Hypertension (HPVG $<10 \mathrm{~mm} \mathrm{Hg}$ ) zu beschränken. In letzter Zeit wurde versucht, die Limits der Operabilität Richtung größere Tumoren zu verschieben: Die 3 wesentlichen Parameter portale Hypertension, Ausmaß der Leberresektion (größer oder kleiner 3 Segmente) und Leberfunktion (MELD-Score) stellen die Eckpfeiler der Risikoabschätzung dar. Eine Resektion von weniger als 3 Lebersegmenten bei einem niedrigen MELD-Score $(\leq 9)$ und Absenz einer klinisch signifikanten portalen Hypertension ist mit einer Mortalität von nur 0,5\% verknüpft [53].

Patienten mit symptomatischer Leberzirrhose haben ein deutlich erhöhtes Risiko bei kardiochirurgischen Eingriffen. Die Mortalität erreicht $25 \%$, die Komplikationsrate $60 \%$. Auch diese Pa- 
tienten sind, wenn verfügbar, mit weniger invasiven therapeutischen Optionen (Stenting, Valvuloplastie) besser versorgt [54].

\section{Notfalleingriffe}

Für Notfalleingriffe ist die Erhöhung von Leberwerten oder die Präsenz einer Lebererkrankung im Wesentlichen irrelevant, so die Operationsindikation und die Dringlichkeit der Operation feststeht. Die Kenntnis einer Lebererkrankung bzw. von erhöhten Leberwerten ist jedoch für die korrekte Interpretation der Laborwerte und des klinischen Verlaufs in der postoperativen Situation von Bedeutung.

\section{Hochrisikosituationen für elektive chirurgische Eingriffe}

Eine Reihe von Hochrisikokonstellationen für elektive Eingriffe lassen sich daher für Patienten mit Lebererkrankung formulieren. Elektive Eingriffe sollten bei akuter und fulminanter $\mathrm{He}$ patitis (Ausnahme: Lebertransplantation zur Therapie der fulminanten Hepatitis), schwerer alkoholischer Hepatitis, schwerer chronischer Hepatitis, Leberzirrhose Child-Pugh B/C, schwerer Koagulopathie und bei extrahepatischen Komplikationen, wie Hypoxie, Kardiomyopathie und akute Niereninsuffizienz, postponiert werden. Für Patienten mit anderer Lebererkrankung soll das Risiko individuell abgeschätzt werden. Im Allgemeinen gilt, dass Patienten mit chronischer Lebererkrankung ohne Zirrhose und Patienten mit ChildPugh-A-Zirrhose extrahepatische operative Eingriffe gut tolerieren und dass auch bei Child-Pugh-B-Zirrhose diese Operationen unter perioperativer Optimierung durchgeführt werden können. In Child-Pugh-B-Zirrhose ist jedoch eine hepatische Resektion, aber auch eine kardiale Operation nur unter Inkaufnahme eines deutlich erhöhten Risikos möglich. Bei Child-Pugh-C-Zirrhose ist von einer elektiven Operation aufgrund der beträchtlichen Mortalität (bis $60 \%$ ) abzuraten.

\section{Präoperative Optimierung}

Einhaltung ethischer Richtlinien

Die Korrektur einer Gerinnungsstörung sollte durch Gabe von Vitamin $\mathrm{K}$ bzw. Blutgerinnungsprodukten auf eine PTZ $>50 \%$, die Thrombozytenzahl auf $>50.000$ gebracht werden. Eine Steuerung der Korrekturmaßnahmen der Blutgerinnung mittels Thromboelastographie verspricht einen reduzierten Einsatz von Fesh-frozen-Plasma und Thrombozytenkonzentraten $[55,56]$. Eine Hypokaliämie, eine Acidose und ein (meist prärenales) Nierenversagen sollte durch entsprechende Elektrolytund Flüssigkeitszufuhr ausgeglichen werden. Aszites sollte mit Nachdruck behandelt werden, um postoperative Wunddehiszenzen und Hernienbildung zu verhindern. Bei Patienten mit Beinödemen kann eine Rekompensation mit Diuretika erfolgen, bei Patienten ohne Ödeme ist eine prä- oder intraoperative komplette Aszitesdrainage von Vorteil. Eine bestehende Therapie der portalen Hypertension mittels nichtselektiven $\beta$-Blockern und einer hepatischen Enzephalopathie mit L-Ornithin-L-Aspartat sollte fortgesetzt werden.

\section{Korrespondenzadresse}

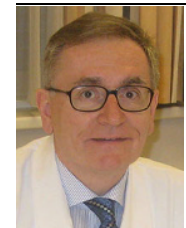

Univ.-Prof. Dr. Christian Müller

Univ.-Klinik für Innere Medizin III, Klin. Abteilung Gastroenterologie und Hepatologie, Medizinische Universität Wien Währinger Gürtel 18-20, 1090 Wien, Österreich christian.j.mueller@ meduniwien.ac.at

Univ.-Prof. Dr. Christian Müller Medizinstudium 1974-1979 an der Universität Wien, 1980-1982 Assistent am Institut für Immunologie Wien, 1982-1987 Ausbildung zum Facharzt für Innere Medizin an der kardiologischen Univ.-Klinik, II. Medizinischen Univ.Klinik, und an der II. Univ.-Klinik für Gastroenterologie und Hepatologie, 1987 FA für Innere Medizin, OA an der II. Univ.-Klinik für Gastroenterologie und Hepatologie, 1990-1991 "research fellow" an der Georgetown University, Washington, DC., Div. of Molecular Virology and Immunology, 1991 Habilitation in innerer Medizin ao. Univ.-Prof. an der Univ.-Klinik für Innere Medizin III, Klin. Abtl. Gastroenterologie und Hepatologie.

Funding. Open access funding provided by Medical University of Vienna.
Interessenkonflikt. C. Müller gibt an, dass kein Interessenkonflikt besteht.

Dieser Beitrag beinhaltet keine von den Autoren durchgeführten Studien an Menschen oder Tieren.

Open Access. Dieser Artikel wird unter der Creative Commons Namensnennung 4.0 International Lizenz (http://creativecommons.org/licenses/by/4.0/deed. de) veröffentlicht, welche die Nutzung, Vervielfältigung, Bearbeitung, Verbreitung und Wiedergabe in jeglichem Medium und Format erlaubt, sofern Sie den/die ursprünglichen Autor(en) und die Quelle ordnungsgemäßnennen, einen Linkzur Creative Commons Lizenz beifügen und angeben, ob Änderungen vorgenommen wurden.

Hinweis des Verlags. Der Verlag bleibt in Hinblick auf geografische Zuordnungen und Gebietsbezeichnungen in veröffentlichten Karten und Institutsadressen neutral.

\section{Literatur}

1. Friedman LS (1999) The risk of surgery in patients with liver disease. Hepatology 29:1617

2. O'Leary JG, Yachimski PS, Friedman LS (2009) Surgery in the patient with liver disease. Clin Liver Dis 13:211

3. Montomoli J, Erichsen R, Christiansen CF et a (2013) Liver disease and 30-day mortality after colorectal cancer surgery: a Danish populationbased cohort study. BMC Gastroenterol 13:66

4. Patel T (1999) Surgery in the patient with liver disease. Mayo Clin Proc 74:593

5. Blum HE (1997) Der Patient mit Hepatopathie. Chirurg 68:763-769

6. Villeneuve JP, Infante-Rivard C, Ampelas $M$ Pomier-Layrargues G, Huet PM, Marleau D (1986) Prognostic value of the aminopyrine breath test in cirrhotic patients. Hepatology 6:928-931

7. Friedman LS, Martin P, Muñoz SJ (1996) Liver function tests and the objective evaluation of the patient with liver disease. In: Zakim D, Boyer TD (Hrsg) Hepatology: a textbook of liver disease, 3. Aufl. Saunders, Philadelphia, S791-802

8. Child CG III, Turcotte JG (1964) Surgery and portal hypertension. In: Child CG III (Hrsg) The liver and portal hypertension. Saunders, Philadelphia, S50

9. Pugh RN, Murray-Lyon IM, Dawson JL et al (1973) Transection of the esophagus for bleeding oesophageal varices. Br J Surg 122:646-649

10. Christensen E, Schlichting P, Fauerholdt L, Gluud C, Anderson P, JuhlE, Poulson Hetal (1984) Prognostic value of Child-Turcotte criteria in medically treated cirrhosis. Hepatology 4:430-435 risk in patients with cirrhosis: from art to science. Gastroenterology 132:1609

12. Malichoc M, Kamath PS, Gordon FD, Peine CJ, Rank J, ter Borg PC (2000) A model to predict poor survival in patients undergoing transjugular intrahepatic portosystemic shunts. Hepatology 31:864-871

13. Wiesner R, Edwards E, Freeman R, Harper A, Kim $R$, Kameth $P$ Kremers W, Lake J, Howard T, Merion RM, Wolfe RA, Krom R, United Network for Organ Sharing Liver Disease Severity Score Committee (2003) The model for end-dtage liver disease
11. O'Leary JG, Friedman LS (2007) Predicting surgical 
(MELD) and allocation of donor livers. Baillieres Clin Gastroenterol 124:91-96

14. Kamath PS, Wiesner RH, Malinchoc M, Kremers W, Therneau TM, Kosberg CL, D'Amico G, Dickson ER, Kim WR (2001) A model to predict survival in patients with end-stage liver disease. Hepatology 33:464-470

15. Mayo Clinic Medical ProfessionalsTransplant Medicine. MELD Model. https://www.mayoclinic. org/medical-professionals/transplant-medicine/ calculators/meld-model/itt-20434705. Zugegriffen: 15.01 .2019

16. Teh SH, Nagorney DM, Stevens SR et al (2007) Risk factors for mortality after surgery in patients with cirrhosis. Baillieres Clin Gastroenterol 132:1261

17. Farnsworth N, Fagan SP, Berger DH, Awad SS (2004) Child-Turcotte-Pugh versus MELD score as a predictor of outcome after elective and emergent surgery in cirrhotic patients. Am J Surg 188:580

18. Perkins $L$, Jeffries $M$, Patel T (2004) Utility of preoperative scores for predicting morbidity after cholecystectomy in patients with cirrhosis. Clin Gastroenterol Hepatol 2:1123

19. Befeler AS, Palmer DE, Hoffman M et al (2005) The safety of intra-abdominal surgery in patients with cirrhosis: model for end-stage liver disease score is superior to Child-Turcotte-Pugh classification in predicting outcome. Arch Surg 140:650

20. Cucchetti A, Ercolani G, Vivarelli M et al (2006) Impact of model for end-stage liver disease (MELD) score on prognosis after hepatectomy for hepatocellular carcinoma on cirrhosis. Liver Transpl 12:966

21. Hanje AJ, Patel T (2007) Preoperative evaluation of patients with liver disease. Nat Clin Pract Gastroenterol Hepatol 4:266

22. Mayo Clinic. Medical ProfessionalsTransplant Medicine. Post-operative Mortality Risk in Patients with Cirrhosis. https://www.mayoclinic.org/medicalprofessionals/transplant-medicine/calculators/ post-operative-mortality-risk-in-patients-withcirrhosis/itt-20434721.Zugegriffen: 15.01.2019

23. Schemel WH (1976) Unexpected hepatic dysfunction found by multiple laboratory screening. Anaesth Analg 55:810-816

24. Wataneeyawech M, Kelly KA Jr (1975) Hepatic diseases unsuspected before surgery. N Y State J Med 75:1278-1282

25. Runyon B (1986) Surgical procedures are well tolerated by patients with asymptomatic chronic hepatitis. J Clin Gastroenterol 8:542-544

26. Behrns KE, Tsiotos GG, DeSouza NF, Krishna MK, Ludwig J, Nagorney DM (1998) Hepatic steatosis is a potential risk factor for major hepatic resection. J Gastrointest Surg 2:292-298

27. Reddy SK, Marsh JW, Varley PR et al (2012) Underlying steatohepatitis, but not simple hepatic steatosis, increases morbidity after liver resection: a case-control study. Hepatology 56:2221

28. Pestana L, Swain J, Dierkhising R et al (2015) Bariatric surgery in patients with cirrhosis with and without portal hypertension: a single-center experience. Mayo Clin Proc 90:209

29. Capussotti L, Polastri R (1998) Operative risks of majorhepaticresections. Hepatogastroenterology 45:184-190

30. Wu CC, Yeh DC, Lin MC et al (2001) Improving operative safety for cirrhotic liver resection. $\mathrm{Br}$ J Surg 88:210

31. Li GZ, Speicher PJ, Lidsky ME et al (2014) Hepatic resection for hepatocellular carcinoma: do contemporary morbidity and mortality rates demand a transition to ablation as first-line treatment? J Am Coll Surg 218:827
32. Berzigotti A, Reig M, Abraldes JG et al (2015) Portal hypertension and the outcome of surgery for hepatocellular carcinoma in compensated cirrhosis: a systematic review and meta-analysis. Hepatology 61:526

33. Garrison RN, Cryer HM, Howard DA, Polk HC Jr. (1984) Clarification of risk factors for abdominal operations in aptients with hepatic cirrhosis. Ann Surg 199:648-655

34. Mansour A, Watson W, Shayani V, Pickleman J (1997) Abdominal operations in patients with cirrhosis: still a major surgical challenge. Surgery 122:730-736

35. Aranha GV, Sontag SJ, Greenlee HB (1982) Cholecystectomy in cirrhotic patients: a formidable operation. Am J Surg 143:55-60

36. Yeh CN, Chen MF, Jan YY (2002) Laparoscopic cholecystectomy in 226 cirrhotic patients. Expe rience of a single center in Taiwan. Surg Endosc 16:1583-1587

37. Quillin RC 3rd, Burns JM, Pineda JA et al (2013) Laparoscopic cholecystectomy in the cirrhotic patient: predictors of outcome. Surgery 153:634

38. Chmielecki DK, Hagopian EJ, Kuo YH et al (2012) Laparoscopic cholecystectomy is the preferred approach in cirrhosis: a nationwide, populationbased study. HPB (Oxford) 14:848

39. Laurence JM, Tran PD, Richardson AJ et al (2012) Laparoscopic or open cholecystectomy in cirrhosis: a systematicreview of outcomes and meta-analysis of randomized trials. HPB (Oxford) 14:153

40. de Goede B, Klitsie PJ, Hagen SM et al (2013) Meta-analysis of laparoscopic versus open cholecystectomy for patients with liver cirrhosis and symptomatic cholecystolithiasis. Br J Surg 100:209

41. Bhangui P, Laurent A, Amathieu R, Azoulay D (2012) Assessment of risk for non-hepatic surgery in cirrhotic patients. J Hepatol 57:874

42. Meunier K, Mucci S, Quentin V et al (2008) Colorectal surgery in cirrhotic patients: assessment of operative morbidity and mortality. Dis Colon Rectum 51:1225

43. Dixon JM, Armstrong CP, Duffy SW et al (1983) factors affecting morbidity and mortality after surgery for obstructive jaundice: a review of 373 patients. Gut 24:845-850

44. McPherson GA, Benjamin Hodgson ISHJ et al (1984) Pre-operative percutaneous transhepatic biliary drainage: the results of a controlled trial. $\mathrm{Br} J$ Surg 71:371-376

45. Lai EC, Mok FP, Fan ST et al (1994) Preoperative endoscopic drainage for malignant obstructive jaundice. Br JSurg 81:1195-1199

46. van der Gaag NA, Rauws EA, van Eijck $\mathrm{CH}$ et al (2010) Preoperative biliary drainage for cancer of the head of the pancreas. N Engl J Med 362:129

47. Diamond T, Park RW (1997) Perioperative management of obstructive jaundice. Br J Surg 84:147-153

48. Fogarty BJ, Parks RW, Rowlands BJ, Diamond T (1995) Renal dysfunction in obstructive jaundice. BrJSurg 82:877

49. Lai EC, MokFP, Tan ESetal (1992) Endoscopicbiliary drainage for severe acute cholangitis. N Engl J Med 326:1582-1586

50. Clavien PA, Petrowsky H, DeOliveira ML, Graf R (2007) Strategies for safer liver surgery and partial liver transplantation. N Engl J Med 356:1545

51. van den Broek MA, Olde Damink SW, Dejong $\mathrm{CH}$ et al (2008) Liver failure after partial hepatic resection: definition, pathophysiology, risk factors and treatment. Liver Int 28:767

52. EASL (2018) Clinical Practice Guidelines: Management of hepatocellular carcinoma European
Association for the Study of the Liver. J Hepatology 69:182-236

53. Citterio D, Facciorusso A, Sposito C, Rota R, Bhoori S, Mazzaferro V (2016) Hierarchic interaction of factors associated with liver decompensationafter resection for hepatocellular carcinoma. JAMA Surg 151:846

54. Gaudino M, Santarelli P, Bruno P et al (1997) Palliative coronary artery surgery in patients with severe noncardiac diseases. Am J Cardiol 80:1351-1356

55. De Pietri L, Bianchini M, Montalti R, De Maria N, Di Maira T, Begliomini B, Gerunda GE, di Benedetto F, Garcia-Tsao G, Villa E (2016) Thrombelastographyguided blood product use before invasive procedures in cirrhosis with severe coagulopathy: a randomized, controlled trial. Hepatology 63:566-573

56. Debernardi Venon W, Ponzo P, Sacco M, Mengozzi G, Raso S, Valpreda A, Rizzetto M, Marzano A (2015) Usefulness of thromboelastometry in predicting the risk of bleeding in cirrhotics who undergo invasive procedures. Eur J Gastroenterol Hepatol 27:1313-1319 